\title{
Formación de la Corporeidad hacia un Desarrollo Integral Corporate training towards integral development
}

\author{
Jayson Bernate, Ingrid Fonseca \\ Corporacion Universitaria Minuto de Dios (Colombia)
}

Resumen. La concepción de cuerpo no ha sido la misma a lo largo de la historia, esta ha sido constante cambiante y transformado debido a las necesidades primarias del ser humano, este a su vez viene instaurando un paradigma para la supervivencia en la sociedad. En los últimos años la Educación Física aporta de manera significativa la formación del ser íntegro holístico, en lugar de la mentalidad deportivista. El objetivo de esta investigación es indagar sobre las tendencias educativas en corporeidad para el desarrollo de una sociedad ética y formal a través de las teorías motrices. La metodología empleada fue una revisión documental-bibliográfica la cual abarcó artículos indexados en las bases de datos como los son Scopus, Dialnet, Sport Discus, WoS, EBSCO, Redalyc entre otras entre los años de 2005-2019. Como principal hallazgo se logró evidenciar que la repercusión de la motricidad y la formación de la expresión corporal en el rol docente, cumple un papel determinante en el ciclo de vida de los seres humanos, ya que influye de manera directa, tanto en el desarrollo motor, como en la concepción epistémica del concepto de cuerpo, generando en los seres humanos conciencia motriz. Como principal conclusión se determina que la formación corporal en todas las etapas de la vida es fundamental para un desarrollo integral en las personas. Palabras clave: Corporeidad, Formación, Educación física, Desarrollo, Motricidad.

\begin{abstract}
The conception of the body has not been the same throughout history, it has been constantly changing and transformed due to the primary needs of the human being, this in turn has been establishing a paradigm for survival in society. In recent years, Physical Education contributes significantly to the formation of the whole holistic being, instead of the sportsmanship mentality. The objective of this research is to investigate the educational trends in corporeity for the development of an ethical and formal society through motor theories. The methodology used was a documentary-bibliographic review which included articles indexed in databases such as Scopus, Dialnet, Sport Discus, WoS, EBSCO, Redalyc, among others, between the years 2005-2019. As the main finding, it was possible to show that the impact of motor skills and the formation of body expression in the teaching role plays a determining role in the life cycle of human beings, since it directly influences both motor development, as in the epistemic conception of the body concept, generating motor consciousness in human beings. As the main conclusion, it is determined that body training in all stages of life is essential for an integral development in people.
\end{abstract}

Keywords: Corporeity, Training, Physical education, Development, Motor skills.

\section{Introducción}

Las diferentes perspectivas e imaginarios que tiene cada persona del cuerpo humano a través de la educación física en el ámbito educativo, pueden asumirse desde la misma formación que han tenido durante la etapa del ciclo vital, a través de prácticas corporales, tendencias en actividad física o simplemente en movimientos físicos del diario vivir. Barbero (2001) parte de la idea que todos los seres humanos tienen un cuerpo, el cual se ha percibido desde la perspectiva holística, integrando la constitución fundamental de cada persona. Sin em-

Fecha recepción: 10-04-20. Fecha de aceptación: 06-08-21 Jayson Bernate

jayson.bernate@uniminuto.edu bargo, a lo largo del tiempo las personas han adoptado la concepción de cuerpo hacia el significado de las ciencias médicas, dando caso omiso a la fisiología o tangibilidad y todos sus derivados. Se tendrá en cuenta que el cuerpo de cada persona es completamente distinto y la forma en que se percibe este y puede concebirse a partir de experiencias vividas en una etapa más temprana de la vida. Una persona al realizar actividad física al interior de una institución educativa, centro deportivo o por autonomía propia, lo lleva a libere hormonas las cuales lo hacen sentir mejor desde el punto de vista fisiológico y automáticamente cambia su expresión corporal, la cual será más segura de sí misma y tranquila, a esto se le llama corporeidad (Águila \& López, 2018), que sería la forma en la cual se perciben las otras personas y la forma en la que quiere expresarse de manera libre. 
La conceptualización del concepto de cuerpo ha sufrido mutaciones a lo largo de la historia, en la edad antigua esta corporeidad se evidenciaba a partir de las capacidades y aptitudes que tenían las personas al llevar sustento a sus grupos familiares, lo cual generaba una imagen frente al resto generando una responsabilidad en ellos mismos frente a su población. Ya en la edad media una joven llamada Helena de Esparta y la revolución que ella lidero fue el comienzo del cuerpo visto como un modelo a seguir, una cultura la cual consistía en el trabajo y cuidado corporal para así tener un mayor nivel en la sociedad.Y, por último, en la modernidad el cuerpo se ha adaptado al modo de tener una buena imagen corporal como un estilo de vida saludable. El principal problema que se logra evidenciar es que en muchos centros educativos, se concibe la educación física desde un punto de vista deportivista y no como una formación de la corporeidad en los niños, para así darles un desarrollo personal y con ello, mejorar su comunicación en un círculo social. (Bermúdez \& Sáenz, 2019)

Es inevitable que al momento de hablar sobre el cuerpo se mencione de una u otra manera la Educación Física, hasta se puede afirmar que muchos de los conceptos acerca de esta materia están definidos a través de varias perspectivas de cuerpo. (Pérez \& Deivis 2003). Entonces acá es donde se logra coincidir en que la Educación Física no solo tendrá en cuenta la concepción de cuerpo, sino que debe lograr la formación integral en los actores de cierta comunidad educativa. Cómo manifiestan Grasso y Erramouspe (2005) desde que se tiene razón el cuerpo se ha visto como un instrumento; el cual debe ser formado, adaptado y constituido para responder a unas necesidades específicas, viendo a este como una máquina la cual se ofrece a los demás por medio de un modelo sistémico y mecanizado. De acuerdo a lo anterior se puede inferir la situación que siempre se ha llevado dentro de la Educación Física, donde los maestros de esta área no se les perciben una formación de sus estudiantes hacia un campo de integralidad y formación de sí mismo, sino que se evidencia en las metodologías un ámbito deportivista y activista.

Por ende las consecuencias de brindar una formación deportivista tendrá como resultado una adquisición equivoca de los estudiantes hacia la construcción conceptual y cognitiva de la teoría de la corporeidad, el agravante de esta situación es que los docentes no se dan cuenta que en estas edades los jóvenes tienen más inseguridades, llevando a una población al no desarrollo del sí mismo, sino de un ser lleno de conocimiento que únicamente se sustenta en lo que ha recibido y no en lo que ha vivenciado. Por ello, entonces la importancia del correcto y óptimo desarrollo de la educación física y el impacto que ella tiene en la sociedad en ámbitos culturales, sociológicos y epistémicos. (Bernate et al. 2019)

Desde luego, la idea de generar un cambio por la corporeidad dentro de la educación física para que así las personas puedan generar un desarrollo social e integral implica una variación ontológica en donde se tiene que evaluar por qué se ha enseñado de una forma u otra, y por qué se debe cambiar esta metodología dentro de esta área del conocimiento. Este cambio debe adoptar un enfoque de endereza el cual trasciende de los dualismos de cuerpo-mente y cuerpo-espacio (Fernández \& Prados, 2014; Fonseca et al. 2019), y se logre identificar cuál será el currículo a seguir al igual que la metodología. A través de del aprendizaje sería necesario fomentar actividades en las cuales se enfoquen hacia el conocimiento de sí mismo y su entorno correspondiente, donde se evidencie la forma en que los estudiantes se vean beneficiados a la hora de adquirir experiencias en todo ámbito posible, cómo lo puede ser, lo corporal, lo social, lo actitudinal, lo cognitivo y, por supuesto, lo emocional, que a su vez tenga impacto dentro del modelo de aprendizaje (Johnson, 2008; Shusterman, 2008). Claramente a la hora que el estudiante desarrolle un sentido propio y consciente de corporeidad logrará elevar y superar el conocimiento personal y tomar las decisiones más adecuadas para el bienestar propio. (Blanco, 2009; Rojas et al. 2019)

Por otro lado la motricidad como lo señalan Backes, Porta \& De Anglat (2015), debe ser parte fundamental del desarrollo humano, en las primeras etapas de vida debido a la capacidad de producir movimientos musculares de manera coordinada y voluntaria en los niños. Es así como se constituyen bases para adquirir el desarrollo del lenguaje y cognitivo del niño; también permite la espontaneidad y creatividad en sus etapas de desarrollo, además de optimizar las habilidades sociales. También Fraile, Tejero, Esteban \& Veiga (2019) mencionan en un trabajo exploratorio y vivencial de la motricidad en edades tempranas, la relevancia del juego y motricidad en el desarrollo en la sociedad mediante unidades didácticas y pedagógicas. De esta forma, se desarrolla de manera integral el niño en todas las dimensiones del ser humano, además ayuda a desarrollar la creatividad, las habilidades motoras, capacidades cognitivas, personales y sociales. Es así como la escuela se convierte en el mejor lugar para socializar y explorar la Educación Física, desarrollando la motricidad en la población infantil. 
Así mismo, Callado (2018) justifica el porqué de la importancia y relevancia de la Educación Física en etapas iniciales del ciclo vital proponiendo los siguientes principios, tales como:

a) La psicomotricidad debe ser el primer aprendizaje motor que se adquiere al empezar a desarrollar en la vida, ya que ayuda al niño en poder utilizar la actividad física como canalizador de su energía, además de involucrar el juego como un factor fundamental para el enriquecimiento de los patrones básicos de movimiento y favorecer sus estados anímicos, sociales y personales.

b) La acción motriz es el motor fundamental para las fases sensibles de desarrollo humano.

c) La educación expresivo-motriz ayuda a descubrir su propio cuerpo por medio de las experiencias y vivencias, lo cual desarrolla el auto cuidado y la autoconfianza, principios inherentes en el desarrollo pedagógico de las sesiones de clase de Educación Física.

d) Esta disciplina ayuda al mejoramiento de la memoria y permite que el niño a poder desarrollar nociones de orientación y espacio en situaciones reales del diario vivir.

Por consiguiente, es relevante mencionar lo descrito por Aristizabal, Ramos \& Chirino (2018) los cuales afirman que la motricidad se debe empezar a desarrollar mientras el niño se divierte. De esta forma, el niño irá perfeccionando sus habilidades motrices, involucrándose más con la socialización y ayude a la creatividad del mismo. Es decir, el juego como eje fundamental desarrolla la motricidad y la socialización en el niño, trae beneficios para mejorar su creatividad, habilidades y cualidades físico-cognitivas, el reconocimiento corporal, y la memoria, etc. De igual forma, la educación motriz es la forma del ser completo, pues asocia la conciencia de la acción, la relación e interacción del sujeto con el entorno, no solo físico sino social y comunitario (Bernate, 2021).

Por otro lado, Garófano \& Guirado (2017) mencionan que:

La motricidad no solo es importante porque permite el desarrollo motor del alumnado, sino también porque a través de ella el niño expresa y comunica sus emociones y adquiere los conocimientos pertenecientes al ciclo, siendo el movimiento un factor determinante en el aprendizaje al ser considerado un agente motivador capaz de impulsar al niño a la acción, que junto al juego ocupa un lugar relevante en el día a día del pequeño. (p. 101)

Por suerte, en años recientes se han propiciado diversas formas de acoplar la Educación física a darle un valor en los procesos de desarrollo para sus practicantes, estos se han inclinado a la mejoría de la práctica pedagógica (Cáceres, 2010; Bernate et al. 2020). Cómo plantean Valencia-Peris \& Lizandra (2018) a pesar que se ha buscado un cambio dentro de este campo, la mejoría ha sido muy lenta, por lo que aún se sigue teniendo una visión tradicional. La causante que no se dé el cambió de una forma óptima, es que desde fuera de la escuela aún se tiene la educación física orientada únicamente hacia la formación motriz y corporal donde se busca formar a los jóvenes para conseguir un rendimiento por sobre su población. (Peña et al. 2018)

Por último, Bernate, Fonseca, Urrea \& Amaya (2019) mencionan la importancia de formar integralmente a los niños desde sus etapas infantiles, fortaleciendo los lazos de habilidades sociales mediante ejercicios y juegos colaborativos, potenciando la educación corporal en valores. Por tal motivo, el objetivo de la siguiente revisión documental está enfocado en revisar los diferentes referentes teóricos y epistémicos, además de las investigaciones en torno a las estrategias pedagógicas utilizadas para desarrollar y potenciar la corporeidad, por medio de la motricidad y educación corporal en diferentes etapas biológicas de los seres humanos.

\section{Metodología}

La metodología de investigación se aplicó a partir de la reflexión, de los referentes conceptuales y epistemológicos, en donde emergen las categorías asociadas en la fase de desarrollo heurística. Desde lo operativo en correspondencia con la dinámica y estrategia de seguimiento y observación, y su respectiva aplicación. Para el desarrollo de la presente fase se implementó una guía, la cual permite ubicar los pasos y acciones; la guía emplea protocolos de búsqueda y revisión de fuentes de información. (Betancourt, Bernate, Fonseca \& Rodríguez, 2020)

El primer protocolo se estructuró en cinco elementos: i) el idioma de las fuentes de información; ii) línea de tiempo; iii) palabras clave; iv) artículos relacionados; v) metodología de estudio. Por su parte, el segundo protocolo se estructuró en cuatro elementos: i) criterios de revisión; ii) los criterios de exclusión (determinados a partir del problema y los intereses del grupo investigador); iii) los criterios de inclusión representados en los tópicos relevantes a la investigación y iv) la estrategia de extracción de datos. En la tabla 1 se aprecia la guía de fase heurística, mostrando aspectos directos de su aplicación. 
Protocolo de fuentes búsqueda de Información

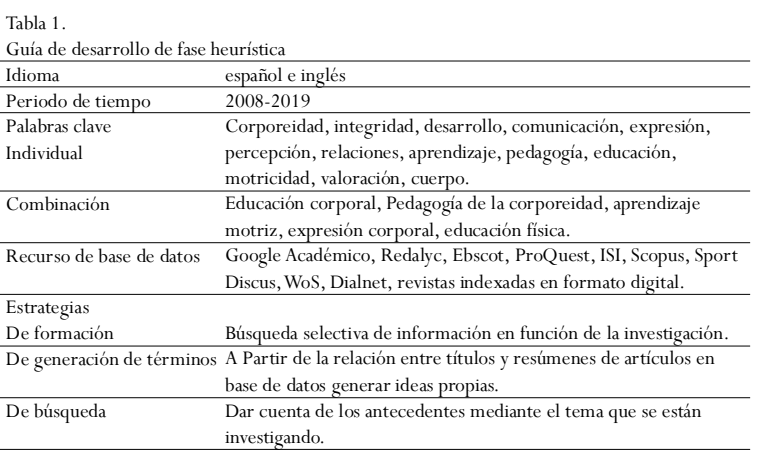

Protocolo de revisión de fuentes de información

Normas de revisión

Rastrear la existencia de trabajos similares al objeto de estudio, problema de investigación, objetivos, autores, leyes, decretos, resoluciones y documentos institucionales. Línea de tiempo ajustados al contexto y referentes institucionales nacionales. Trabajos publicados en revistas indexadas y revistas científicas arbitradas especializadas. Hacer lectura del resumen e introducción como estrategia de inclusión o exclusión preliminar.

\section{Criterios de Exclusión}

Publicaciones que no están relacionadas con el contexto específico del objeto de estudio. Publicaciones que no se encuentren en revistas científicas, indexadas, bases de datos y referenciadas como productos no investigativos. Publicaciones que se encuentren fuera de la línea de tiempo, establecida en el presente estudio.

\section{Criterios de inclusión}

Palabras clave referenciadas. Abordaje del diseño de investigación, problema de investigación, metodología, y referentes conceptuales. Publicaciones que se encuentren en la línea de tiempo establecida para la investigación.

\section{Evaluación de los hallazgos encontrados}

Para dar cuenta de la aplicación de un protocolo para la revisión de fuentes de información, se construyó una matriz de recolección de datos, en donde se da cuenta de los criterios de inclusión de la investigación. A continuación, se presenta la estructura del formato de revisión documental, el cual evidencia los tópicos más significativos: título de la publicación, fecha de publicación, autores, diseño, muestra o instrumentos, objetivo, metodología y principal aporte. (Ver Tabla 2)

\section{Resultados}

Ver tabla 2.

\section{Discusión}

Los resultados están en relación con los documentos consultados, a través de la revisión bibliográfica, se observa que el desarrollo y repercusión que tienen las personas adultas en la sociedad tiene mucho que ver en la formación de la primera infancia, ya que en esta etapa se desarrollan los procesos locomotores a través de experiencias de aprendizaje corporales, que a su vez alimentaran la personalidad y el enfoque integral del concepto de persona holística, por ende es pertinente conceptualizar la Educación Física como una herramienta humana para formar seres en corporeidad y motricidad.

En el caso de Parsons (2009) menciona que a medida que los infantes van creciendo a lo largo de su vida, es imperante formar y desarrollar la concepción epistemológica del concepto de cuerpo, ya que va a ser el principal cimiento para que desarrolle su estadio de locomoción. Este desarrollo de las capacidades no se da solo sino que hay que irlo trabajando continuamente, para esto se tiene una clasificación de las edades sensibles en donde los infantes deben estimular la capacidad de acuerdo a sus edades y experiencias que se obtienen con el tiempo. Esto se da para que de esta forma se pueda optimizar mucho más el trabajo realizado, ya que si se enseña un aprendizaje en una edad o etapa que no pertenezca al niño causará un conflicto fisiológico y morfológico en sus patrones básicos de movimiento, llegando a descartar el progreso que se había ganado con anterioridad.

Según García (2015) para hablar del desarrollo físico se debe trazar una línea en la cual se exponga el crecimiento de la masa corporal en lo individuos, también se tiene en cuenta los demás parámetros como la ingesta de alimentos proporcionando esto un cambio fisiológico dentro del individuo. Están maduración en un proceso que a lo largo del tiempo se ve reforzado y es la principal fuente de cambio, ya que proporciona a las partes del cuerpo las sustancias necesarias para estos metabolismos. Por consiguiente se deben valorar la magnitud y calidad de los cambios para poder comprender la forma de actuar en las diferentes etapas de la vida. Al darse una actividad física integral se dan mejoras en todos los campos posibles hasta el momento de verse el fruto de un aprendizaje motor óptimamente impartido en el sistema locomotor. 
Tabla 2.

Matriz de Análisis Documental

\begin{tabular}{|c|c|c|c|}
\hline Autor/Título & Método/Objetivo & Diseño & Muestra/Instrumentos \\
\hline Toro (2010). Corporeidad y & Identificar la importancia que tiene una sana & Estudio de & 3 niños diarios de campo y guion de observación \\
\hline $\begin{array}{l}\text { lenguaje: la acción como texto y } \\
\text { expresión. }\end{array}$ & $\begin{array}{l}\text { formación de la corporeidad y su repercusión frente } \\
\text { al lenguaje de cada individuo. }\end{array}$ & caso & \\
\hline $\begin{array}{l}\text { Gonzales \& Gonzales (2010) } \\
\text { Educación física desde la } \\
\text { corporeidad y la motricidad. }\end{array}$ & $\begin{array}{l}\text { Se buscó dar respuesta al cómo los niños a partir de } \\
\text { experiencias en sus edades tempranas forman un } \\
\text { carácter y así afrontan sus relaciones y actitud frente } \\
\text { a las actividades escolares }\end{array}$ & $\begin{array}{l}\text { Narrativas } \\
\text { Reflexivas }\end{array}$ & $\begin{array}{l}40 \text { Niños y } 35 \text { Niñas de edades escolares, formato de } \\
\text { análisis de narrativas y sistematización de } \\
\text { experiencias pedagógicas. }\end{array}$ \\
\hline
\end{tabular}

Aportaciones/Hallazgos

Trol docente tiene bastante importancia dentro el desarrollo de cada persona y para suplir esta problemática
puede ajustar actividades en donde se evidencian diferentes manifestaciones cómo podría ser la danza y el baile.

Debate dialogico en la formacion de los estudiantes con la metodología que cada docente, la cual se adquirió mediant los principios pedagógicos de educación corporal infantil, creando un modelo pedagógico basado en las experiencias de sí mismo y su interacción con la danza y la pedagogía motriz corporal

Ferrada (2009) Implementar una propuesta pedagógica innovadora, Estudio de 1 niño, formato de diario de campo, guion de Creación de un modr

El principio de emocionalidad
corporeidad: un complemento a través de la educación corporal para dar solución a caso corporeidad: un complemento al problemas que se presentan en la cotidianidad, como psicológica.

basado en vive uncelo experimental de aprendizaje m modificando el patrón de aprendizaje inicial en educacis

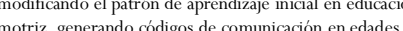
lo son; malos hábitos alimenticios, vicios posturale, enfermedades no trasmisibles y problemas de convivencia escolar:

García et, al. (2016) Actividades Consiste en diseñar un grupo de actividades físico- Cualitativa físico-recreativas para el desarrollo deportivas que influya directa e indirectamente en el motriz en niños de 8-10 años de la desarrollo motriz de niños entre los 8-10 años de escuela rural 'Ciudad de Riobamba', edad.

recinto Santa Lucia, parroquia

Camarones del Cantón Esmeraldas,

Ecuador

Gaviria et, al. (2019) ¿Qué aprende El objetivo del estudio fue estudiar las creencias y $\quad$ Mixta el estudiantado de secundaria en la percepciones del alumnado relativas al aprendizaje asignatura de educación física? en las clases de educación física. iniciales basados desde el modelo de corporeidad.

70 niños y niñas de edades entre 8 y 10 años, 50\% se diagnosticaron cualitativamente los gustos de los niños del grupo masculino y el otro $50 \%$ femenino. Los para de esta forma, realizar las actividades pertinentes a sus niños fueron puestos a prueba por un promotor intereses y necesidades de acuerdo a la sociedad que deportivo, líder del consejo comunal, vecinos y pertenezcan. padre de familia para hacer constancia del progreso en la actitud de los niños a lo largo del proyecto. y se utilizaron la entrevista y el diario de campo. Participaròn 93 estudiantes de grado 11, de una cómo principal hallazgo muestran que los estudiantes institución educativa de la ciudad de Medellín- enmarcan que en dicha institución, que el aprendizaje de la Colombia. La información se recolectó por medio enmacáón física se basa principalmente, hacia la parte motriz de cuestionario, observación y grupos focales, para dejando de lado actividades que les brinden un desarrollo el análisis de la información se utilizó el software social y cultural. el analisis de la informacion se utilizo el software
spss para la información del cuestionario y el spss para la informacion del cuestionario y
programa nvivo para los grupos focales y la observación

\begin{tabular}{ll}
\hline $\begin{array}{l}\text { Ruiz et, al. (2017) Evaluar la } \\
\text { Coordinación Motriz Global en }\end{array}$ & $\begin{array}{l}\text { El objetivo de este estudio fue el desarrollo y } \\
\text { evaluación métrica del test motor Sport-Com }\end{array}$
\end{tabular} Coordinación Motriz Global en evaluación métrica del test motor Sport-Comp,
Educación Secundaria: ElTest Motorinstrumento diseñado para ayudar a los profesore Educación Secundaria: El Test Motorinstrumento diseñado para ayudar a los pro
Sport-Comp educación física en la evaluación de la de educación física en la evaluación de la
coordinación motriz global de sus alumnos de educación secundaria $\begin{array}{ll}\text { Cuantitativa } & \begin{array}{l}\text { El presente test se construyó a partir de una revisi } \\ \text { de la literatura científica sobre medición motriz } \\ \text { entre los } 12 \text { y } 17 \text { años. La validez de contenido de }\end{array}\end{array}$ entre los 12 y 17 años. La validez de contenido de las pruebas empleadas fue evaluada por expertos y las pruebas seleccionadas fueron aplicadas a 5732 satisfactorias y ofrecen buenas posibilidades para ser empleado por los profesores de educación física en sus clases por su bajo coste económico, poco tiempo de aplicación escolares de estas edades.

reclamado y poseer normas ajustadas por edad y sexo. Así detectar a los alumnos con sospecha de poseer problemas de coordinación motriz y por lo tanto contribuir a la mejora de los programas de educación física que palien esta condición.

Sailema et, al (2019) Juegos tradicionales como estimulador motriz en niños con sindrome Down

El objetivo de la investigación es realizar una estimulación motriz efectiva a través del uso de juegos tradicionales ecuatorianos, potenciando destrezas motoras, locomotrices, no locomotrices de proyección, valorado el efecto producido.

Salazar \& Calero (2019) Influencia Analizar la influencia de un programa de actividad Cuantitativa de la actividad física en la física específica para la motricidad fina y gruesa del motricidad fina y gruesa del adulto adulto mayor del sexo femenino, comparando las mavor femenino influencias ejercidas en un grupo con participación sistemática y otro grupo con participación limitada.

\begin{tabular}{ll}
\hline Alonso \& Pazos (2020) Importancia & $\begin{array}{l}\text { Pretendieron aproximarse al conocimiento de la } \\
\text { percibida de la motricidad en }\end{array}$ \\
$\begin{array}{ll}\text { Educación Infantil en los concreta de la práctica motriz y averiguar } \\
\text { educativos de Vigo (España) }\end{array}$ & $\begin{array}{l}\text { qué importancia se le da a la misma en el segundo } \\
\text { ciclo de educación infantil en las escuelas del } \\
\text { ayuntamiento de Vigo, España. }\end{array}$
\end{tabular}

\begin{tabular}{|c|c|}
\hline $\begin{array}{l}\text { Pérez (2017) Habilidades del área } \\
\text { motriz fina y las actividades de } \\
\text { estimulación temprana }\end{array}$ & $\begin{array}{l}\text { Potencializar, corregir y prevenir cualquier } \\
\text { alteración en el desarrollo integral de los niños y } \\
\text { niñas que asisten al centro de desarrollo del buen } \\
\text { vivir "San Luis". }\end{array}$ \\
\hline $\begin{array}{l}\text { Águila \& López (2018) Cuerpo, } \\
\text { corporeidad y educación: una } \\
\text { mirada reflexiva desde la Educación } \\
\text { Física }\end{array}$ & $\begin{array}{l}\text { Mostrar y comprender la importancia de } \\
\text { 'incorporar' los posibles progresos teóricos y } \\
\text { prácticos sobre corporeidad, con la finalidad de } \\
\text { lograr un acercamiento hacia una educación integral } \\
\text { y holística, así como romper con los enfoques más } \\
\text { tradicionales del cuerpo que siguen instaurados en } \\
\text { los ámbitos educativos y reproducidos a través de la } \\
\text { educación física. }\end{array}$ \\
\hline
\end{tabular}
programa de actividad físca integral de un programa de educación fisica en donde programa de actividad fisica integral de un programa de educacion fisica en donde el
sobre la motricidad gruesa de niños alumnado sea formado en un estado de plenitud y sobre la motricidad gruesa de ninos alumnado sea formado en un esta
y niñas con diversidad funcional concordancia a sus necesidades.

Choi et, al. (2018) El desarrollo de Realizar u estudio clínico sobre la habilidades habilidades motoras finas es asociado con el lenguaje expresivo resultados en lactantes con alto $y$ bajo riesgo de desorden del espectro autista motoras en bebes con síndrome de TEA.

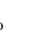

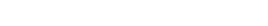

Hurtado (2008) Corporeidad y Reflexionar sobre la teoría del concepto de

motricidad. Una forma de mirar los corporeidad y su aporte a la motricidad hum desde la mirada de los saberes corporales

\section{Cuantitativa}

5 niños portadores del síndrome de Down entre $5-\mathrm{A}$ 15 años de edad, evaluando diferentes habilidades motrices antes y después de implementada la propuesta a partir del test de bruininks-ozeretzki mediante tres criterios (adquirido, en vías de admisión y no adquirido), aplicando la propuesta
lúdica durante seis meses en 72 sesiones de $40 \mathrm{~min}$, tres veces por semana.

Dos grupos independientes de 34 mujeres entre 60 a 80 años, con un nivel socioeconómico medio, residentes en selva alegre - sangolquí. Se aplicó durante cuatro meses un programa especializados actividad física, implementando diez actividades cotidianas con mayor magnitud de psicomotricidad fina y diez de psicomotricidad gruesa, evaluándose en dos momentos el rendimiento motriz bajo una escala entre 1-5 puntos.

Al inicio de la realización de la propuesta se evidencian

varias carencias en la eficiencia motriz y cognitiva de los
niños, pero a medida que se va desarrollando la actividad se evidenciaba una excitación motriz por parte de los estudiantes, lo cual generaba un desarrollo como aprendizaje significativo.

Se logra concluir que dentro del grupo de adultos mayores las mujeres son quienes se pueden desempeñar mucho más sobre los hombres en el tema de la motricidad.

43 escuelas de Vigo, el $50 \%$ del total, obteniendo una muestra total de cincuenta cuestionarios, los importide de los profesionales consideraban que es

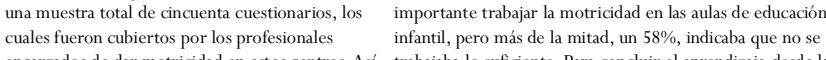
encargados de dar motricidad en estos centros. Así trabajaba lo suficiente. Para concluir el aprendizaje desde la mismo, se han analizado las memorias verifica de las educación física sirve como la base fundamental de ahí en universidades gallegas y el decreto 330/2009 de adelante para lograr sobresalir en los demás campos educación infantil.

Sistematizació Se trabajó con niños en las edades entre 1 a 2 años, 2 Después de ver los resultados se llegó a la conclusión de que a 3 años y 3 a 4 años teniendo en cuenta los su nivel de motricidad fina están muy por debajo del nivel $\begin{array}{lll}\mathrm{n} \text { de } & \text { a } 3 \text { años y } 3 \text { a } 4 \text { años teniendo en cuenta los } & \text { su nivel de motricidad fina están muy por debajo del nivel } \\ \text { experiencias } & \text { diagnósticos determinados para cada edad, para un } & \text { normal, estos factores pueden ser por la situación de sus }\end{array}$ $\begin{array}{lll}\text { experiencias } & \text { diagnósticos determinados para cada edad, para un normal, estos factores pueden ser por la situación de sus } \\ \text { motrices. } & \text { total de } 40 \text { niños y se aplicó un diario de campo, } & \text { hogares, personas quienes los cuidan, cultural, estrato social, }\end{array}$ $\begin{array}{ll}\text { entrevista y guion de observación. } & \text { etc., llevando a una enseñanza totalmente desubicada de un }\end{array}$ $\begin{array}{ll}\text { entrevista y guion de observación. } & \begin{array}{l}\text { etc. llevando a una enseñanza totalmente desubical } \\ \text { ser integral en la dimensión motriz y axiológica. }\end{array}\end{array}$ Revisión $\quad$ 2007-2009 -bases de datos de libre acceso (google $\quad$ Determinar que nunca se ha tenido un concepto estable de documental académico, Redalyc, redib, Dialnet, SciELO DOAJ, cuerpo sino que con el pasar de los años su mirada ha ido revista indexas en SCOPUS) cambiando, viéndolo tanto como un instrumento para la supervivencia como modelo para ventas en un sistema capitalista.

\begin{tabular}{|c|c|c|}
\hline Cuasi- & Se realizó un test el cual media la motricidad gruesa & Se afirmó que se cumple la hipótesis planteada y se ofrece \\
\hline $\begin{array}{l}\text { Experimental } \\
\text { tomando } \\
\text { medidas pre- } \\
\text { test y post- } \\
\text { test. }\end{array}$ & $\begin{array}{l}\text {, de los estudiantes, se logra ver que a medida que el } \\
\text { test avanzaba el desempeño de los jóvenes iba } \\
\text { mejorando. Implementando el test Gross Motor } \\
\text { Function Measure (GMFM } 88 \text { ) y dos encuestas de } \\
\text { valoración personal. A } 12 \text { niños de } 5 \text { a } 12 \text { años. }\end{array}$ & $\begin{array}{l}\text { una amplia respuesta a la pregunta de investigación sugerida, } \\
\text { puesto que el programa de actividad física integral provocó } \\
\text { mejoras significativas en la motricidad gruesa de los niños/as } \\
\text { escolarizados con diversidad funcional. }\end{array}$ \\
\hline $\begin{array}{l}\text { Inter vención } \\
\text { clínica }\end{array}$ & $\begin{array}{l}71 \text { nacidos de alto riesgo con diagnóstico de TEA se } \\
\text { evaluaron las habilidades motoras finas a los } 6,12, \\
18 \text { y } 24 \text { meses de edad y los resultados del lenguaje } \\
\text { expresivo a los } 36 \text { meses usando diferentes test } \\
\text { clínicos médicos. }\end{array}$ & $\begin{array}{l}\text { Generar y propiciar las herramientas necesarias para el } \\
\text { correcto aprendizaje de los bebés en estado de riesgo, el } \\
\text { objeto a desarrollar se complica mucho más pero esto se } \\
\text { puede concluir con una enseñanza dedicada y paciente, a } \\
\text { niveles de motricidad infantil generando estímulos a nivel } \\
\text { cerebral mediante propuestas de acciones motoras } \\
\text { continuas, mejorando el ámbito locomotor en los pacientes. }\end{array}$ \\
\hline $\begin{array}{l}\text { Reflexión } \\
\text { epistémica }\end{array}$ & $\begin{array}{l}\text { Referentes teóricos y epistémicos de la teoría en } \\
\text { corporeidad }\end{array}$ & $\begin{array}{l}\text { Corporeidad y motricidad no son simples distinciones } \\
\text { utilizadas para refinar el lenguaje, son dos conceptos de } \\
\text { tradición fenomenológica que han empezado a ser utilizados } \\
\text { en los últimos tiempos en el contexto de una nueva "ciencia" } \\
\text { llamada motricidad humana. }\end{array}$ \\
\hline
\end{tabular}


Por otro lado, teniendo en cuenta todos los ítems del test Gross Motor Function Measure (Medida de la función motora gruesa) la cual es una herramienta de evaluación diseñada y evaluada para medir los cambios en la función motora gruesa a lo largo del tiempo o con intervención en niños con parálisis cerebral, esto se pone en discusión ya que (Da Fonseca, 2002; Bernate et al. 2020) dan a entender que cuando las personas son sometidas a un modelo de evaluación toman más responsabilidad sobre lo que hacen, y esto se puede ver en todos los ámbitos de la vida, cuando la acción que se debe realizar, está siendo supervisada y tendrá una evaluación devolutiva siempre dando el máximo de cada uno. En este momento también interviene la psicomotricidad en donde cada persona realiza sus movimientos a partir de la razón, apoyándose en esta para a través de una experiencia adquirida anteriormente es implementada en caso de necesitarla. Igualmente, la educación psicomotriz tiene que ajustar a las necesidades que presente tanto en el niño cómo el ambiente sociocultural en el que se encuentra, porque en este momento este concepto no solo afectará positiva o negativamente al niño sino que será una cadena en la que cualquier persona dentro de su círculo social también tendrá repercusiones. (Cameselle, 2005; Bernate et al. 2020).

Por consiguiente, se puede inferir que la psicomotricidad tiene un factor fundamental para la formación en una corporeidad integra, puesto que puede ayudar a todos a comprender y mejorar las relaciones con sí mismo y a su vez con las personas que nos rodean y toda relación con el entorno. La psicomotricidad tiene su auge en el ser humano a la edad de la primera infancia, donde le brinda al niño un desarrollo motor, expresión corporal y producción de conocimientos a través del cuerpo, a medida que el niño va creciendo mejora sus capacidades por el simple hecho de tener una razón sobre los movimientos, el cual genera una imagen de sí mismo, comprende el mundo, establece relaciones con las demás personas.

Esta psicomotricidad vendría siendo la base fundamental para cualquier desarrollo dentro del niño en donde formará su personalidad y le dará reconocimiento para ser una persona autónoma (Pacheco, 2015; Bernate, 2021) Hasta el momento siempre se ha venido hablando sobre un desarrollo en la primera infancia pero ¿ y los adultos mayores qué ?, si bien la calidad de vida es un aspecto subjetivo que cada quien, es dependiendo de sus vivencias y tiene unos parámetros los cuales, definen si se está teniendo un envejecimiento satis- factorio o dramático, acá interviene la actividad física enfocada desde el punto de vista corpóreo, donde los adultos mayores vuelven a generar esa imagen de fortaleza, confianza e interés por ellos mismos, teniendo como resultado una persona más segura y sana tanto física como mentalmente. (Gonzales, 2015; Bernate y Vargas, 2020)

También, se encuentra que el juego es una estrategia o recurso de desarrollo motriz, el cual utilizan los docentes de Educación Física para empezar a desarrollar las habilidades motrices de los niños, pues es así como empiezan el reconocimiento y experimentación de sí mismos con base a su entorno, enriqueciendo su imaginación y sus interacciones sociales, no solo se centra en estos puntos, también en fundamentar la confianza de sí mismo y de los que lo rodean. No obstante, Gómez (2017) determina algunos resultados para que el juego logre ser utilizado como una estrategia didáctica, mencionando que este debe potencializar los procesos de aprendizaje autónomo y cooperativo, de manera en que los docentes implementen estrategias para fortalecer el trabajo de ambos aprendizajes, el ambiente debe ser idóneo para la participación activa y motivacional de los infantes, permitiéndoles aprender uno del otro, aprender a integrarse en grupos y construir modos de auto conocimiento.

En efecto Mori (2008) menciona que la coordinación y motricidad tienen repercusión sobre la memoria de manera directa siendo un aspecto imprescindible a trabajar en los procesos motrices infantil, En consecuencia Urrea et, al. (2019) Hablan de la repercusión que tiene la motricidad y formación de la expresión corporal en el rol docente, donde el maestro tiene el rol más importante ya que es un guía sobre los estudiante, desde ese lugar él va ayudando a construir la forma de expresión corporal y verbal de su clase, es donde aparte de brindarles conocimiento debe analizar, leer y detallar el contexto en el cual se encuentran para brindar los aprendizajes pertinentes. Por tal motivo, el maestro debe formar de igual manera su imagen corporal al momento de ser observado por sus estudiantes y esto tiene una llegada tanto de acercamiento o distanciamiento sobre su clase. Esto se ve gratificado por un buen desarrollo en la infancia, puesto que si una persona es instruida adecuadamente de forma integral genera sobre las demás personas imagen de seguridad y confianza lo cual es indispensable para el rol mismo del ser humano en cualquier tipo de contexto.

Finalmente, no todo lo puede cumplir el docente, es ahí donde la familia entra a jugar un papel fundamental, 
pues este debe ser un mecanismo de ayuda para que el niño encuentre apoyo y consejos de cómo mejorar en cada sesión de trabajo motriz; esto quiere decir ayudar al niño emocionalmente con valores, normas, aprender acatar órdenes, brindarle espacios apropiados para explorar o desarrollar reconocimiento mediante el juego, cabe resaltar que los padres empiezan a brindar procesos de enseñanza desde la etapa prenatal, mediante pequeños procesos de movimientos. (Mamani et al. 2020)

\section{Conclusiones}

Se puede concluir que la base fundamental para un desarrollo integro en los individuos sería una correcta línea de aprendizaje de la educación física, y no ver al cuerpo únicamente como una estructura fisiológica sino también se tiene en cuenta su desarrollo cognitivo. De esta manera se formará una persona tanto motrizmente capaz, como cognitivamente suficiente para afrontar cualquier circunstancia que se le presente.

De igual manera se puede inferir que una persona forma una perspectiva de sí mismo de acuerdo a sus capacidades las cuales han sido adquirida por vivencias que cada persona desarrolla de distinta forma, aquí es donde surge el que cada persona se destaque y se enfoque hacia ámbitos que lo representan y rodearse de un círculo social que se acople a su personalidad. Por tanto, la acción docente se centra en crear experiencias corporales vividas con atención plena, observando las emociones, pensamientos y sensaciones físicas, para, posteriormente, reflexionar sobre las mismas y su transferencia a situaciones de la vida cotidiana. Así, se puede integrar lo vivido corporalmente, con lo sentido emocionalmente y los aspectos cognitivos implicados en la tarea de una manera significativa. Asimismo, trabajar desde la corporeidad supone favorecer y priorizar el ejercicio de la creatividad, la innovación, la expresión y la comunicación en cualquier dominio de acción no sólo en las actividades artísticas expresivas de manera específica, pues la persona está en el centro de la experiencia, es la protagonista y creadora de su corporeidad.

La manifestación de la motricidad, se evidencia en instrumentos que permiten la facilidad por la cual el niño empieza a demostrar desarrollo de sus habilidades motoras y las cuales deben apoderarse de todos los espacios en los cuales se encuentren. Luego, la intervención de los docentes y familiares para el proceso de desarrollo, brindando las herramientas y apoyos para que estos puedan tener un mejor desempeño según lo que la motricidad implica en todos sus ámbitos ya mencionados. Con esto, se busca enriquecer, estimular la importancia de la motricidad en edades tempranas y su gran conexión con las demás áreas del aula y sociales del entorno que los rodean.

Con base a los resultados expuestos dentro de la mayoría de artículos consultados nos expone que una educación física integral tiene repercusiones tanto para la persona que esté recibiendo ese conocimiento como para la sociedad o contexto que lo rodea. Esta responsabilidad cae en el docente, él debe tener en cuenta la importancia de su labor, en donde si por dichas circunstancias hace mala práctica de su cargo puede llegar a dañar un ser y su alrededor. Por eso la educación física debería dejarse de ver cómo un espacio deportivista y de tiempo libre, tanto (docente- estudiante). A la hora de llegar a un acuerdo de las dos partes la clase tomará otro sentido en donde el estudiante acepta que esta es su formación y tendrá claro que es un bienestar consigo mismo y por otro lado el maestro bajo sus criterios y el análisis de su población brindara los aprendizajes que más se adapten a las necesidades de su clase.

\section{Referencias}

Águila Soto, C., \& LópezVargas, J. (2018). Cuerpo, corporeidad y educación: una mirada reflexiva desde la Educación Física (Body, corporeity and education: a reflexive view from Physical Education). Retos, O(35), 413-421. Recuperado de https: / / recyt.fecyt.es/index.php/retos/article/view/62035/ 41748

Alonso Álvarez, Yolanda, \& Pazos Couto, José María. (2020). Importancia percibida de la motricidad en Educación Infantil en los centros educativos de Vigo (España). Educação e Pesquisa, 46, e207294. Epub January 20, 2020.https: / /dx.doi.org/ 10.1590/s1678-4634202046207294

Backes, B. M., Porta, M. E., \& de Anglat, H. E. D. (2015). El movimiento corporal en la educación infantily la adquisición de saberes. Educere, 19(64), 777-790. https:// www.redalyc.org/pdf/356/35643544010.pdf

Barbero, J.I. (2001). Cultura corporal: ¿Tenemos algo que decir desde la Educación Física? Ágora para la Educación Física y el Deporte, 1, 18-36

Bernate, J. (2021). Educación Física y su contribución al desarrollo integral de la motricidad/Physical Education and its contribution to the comprehensive development of motor skills. PODIUM - Revista de Ciencia y Tecnología en la Cultura Física, 16(2), 643-661. Recuperado de https:// podium.upr.edu.cu/index.php/podium/article/view/957 Bernate, J. (2021). Pedagogía y Didáctica de la Corporeidad. Una mirada desde la praxis (Pedagogy and Didactics of Corporeality. A look from praxis). Retos, 42, 27-36. https://doi.org/ 
10.47197/retos.v42i0.86667

Bernate, J., Bejarano, B., \& Cardozo, D. (2020). Cotejo de las competencias ciudadanas en estudiantes de Licenciatura en Educación Física. Mendive. Revista de Educación, 18(3), 647660.

Bernate, J., Fonseca, I., Jiménez, M. J. B., \& Romero, E. (2020). Análisis de las competencias ciudadanas en estudiantes de Licenciatura en Educación Física. PODIUM: Revista de Ciencia y Tecnología en la Cultura Física, 15(2), 202-220.

Bernate, J., Fonseca, I., \& Betancourt, M. (2019). Impacto de la actividad física y la práctica deportiva en el contexto social de la educación superior (Impact of physical activity and sports practice in the social context of higher education). Retos, 37(37), 742-747. Recuperado de https:/ / recyt.fecyt.es/index.php/ retos/article/view/67875/48883

Bernate, J., Fonseca, I., \& Urrea, P. (2019). Impacto en los procesos de la internacionalización y transnacionalización en la educación física superior UNIMINUTO. EmásF; revista digital de educación física, (61), 11-23.

Bernate, J., Fonseca, I., Urrea, P., \&Amaya, G. (2019). La educación física y el deporte social para la paz y el posconflicto en Colombia. EmásF; revista digital de educación física, (61), 33-47.

Bernate, J., Guataquira, A., Rodríguez, J., Fonseca, I., Rodríguez, M., \& Betancourt, M. (2020). Impacto académico y profesional de un programa de educación física a nivel universitario (Academic and professional impact of university physical education program). Retos, (39), 509-515. https://doi.org/ 10.47197/retos.v0i39.81087

Bernate, J., \& Vargas Guativa, J. (2020). Desafíos y tendencias del siglo XXI en la educación superior / Challenges and trends of the 21st century in higher education. Revista de Ciencias Sociales, 26, 141-154. Doi:http://dx.doi.org/10.31876/ rcs.v26i0.34119

Bermúdez Torres, C., \& Sáenz-López, P. (2019). Emociones en Educación Física. Una revisión bibliográfica (2015-2017) (Emotions in Physical Education. A bibliographic review (2015-2017)). Retos, 36(36), 597-603. Recuperado de https: / / recyt.fecyt.es/index.php/retos/article/view/ 70447

Betancourt, M., Bernate, J., Fonseca, I., \& Rodríguez, L. (2020). Revisión documental de estrategias pedagógicas utilizadas en el área de la educación física, para fortalecer las competencias ciudadanas (Documentary review of pedagogical strategies used in the área of physical education to strengthen citizen competenci. Retos, 38(38), 845-851. Recuperado de https:/ /recyt.fecyt.es/index.php/retos/article/view/74918/ 49523

Blanco Vega, M. (2009). Enfoques teóricos sobre la expresión corporal como medio de formación y comunicación. Horizontes Pedagógicos, 11(1). Recuperado a partir de https:// horizontespedagogicos.ibero.edu.co/article/view/332

Cáceres, M. (2010). La expresión corporal, el gesto y el movimiento en la edad infantil. Revista digital para profesionales de la enseñanza, 9(1), 1-7. Recuperado de: https:// www.feandalucia.ccoo.es/docu/p5sd7343.pdf

Callado, C. V. (2018). El aprendizaje cooperativo en educación física: Planteamientos teóricos y puesta en práctica. Acción motriz, (20), 7-16. https://dialnet.unirioja.es/servlet / articulo?codigo $=6435704$

Cameselle, R. P. (2005). Psicomotricidad:Teoría y praxis del desarrollo psicomotor en la infancia. Ideaspropias Editorial SL.

Choi, B., Leech, K.A., Tager-Flusberg, H. et al (2018). Development of fine motor skills is associated with expressive language outcomes in infants at high and low risk for autism spectrum disorder. J Neurodevelop Disord 10, 14 https://doi.org/10.1186/s11689-018-9231-3

Da Fonseca, V. (2002). El cuerpo de la cultura y la cultura del cuerpo. Psicomotricidad: Revista de estudios y experiencias, (70), 17-27.

Fernández-Balboa,J.M., \& Prados Megías, E. (2014).The conscious system for the movement technique: an ontological and holistic alternative for (Spanish) physical education in troubled times. Sport, Education and Society, 19(8), 1089-1106.

Ferrada, D. (2009). El principio de emocionalidad-Corporeidad: Un complemento al modelo de aprendizaje ideológico. Educere, 13(44), 29-38.

Fonseca, I., Bernate, J., Betancourt, M., Barón, B., \& Cobo, J. (2019). Developing Social Responsibility in University Students. In Proceedings of the 2019 11th International Conference on Education Technology and Computers (ICETC 2019). Association for Computing Machinery, NewYork, NY, USA, 215-218. Doi: https://doi.org/10.1145/ 3369255.3369275

Fraile García, J., Tejero-González, C. M., Esteban-Cornejo, I., \& Veiga, Óscar L. (2019). Asociación entre disfrute, autoeficacia motriz, actividad física y rendimiento académico en educación física (Association between enjoyment, motor self-efficacy, physical activity and academic performance in physical education). Retos, 36(36), 58-63. https://doi.org/10.47197/ retos.v36i36.63035

García, V., Calero, S., Chávez, E. \& Bañol, C. (2016). Actividades físico-recreativas para el desarrollo motriz en niños de 8-10 años de la escuela rural 'Ciudad de Riobamba', recinto Santa Lucia, parroquia Camarones del Cantón Esmeraldas, Ecuador. Revista E.F. Deportes.com, Numero. 212. Recuperado de: https: / / www.efdeportes.com/efd212/desarrollo-motrizen-la-escuela-rural.htm

García-Peñalvo, F. J. (2015). Mapa de tendencias en Innovación Educativa. Education in the knowledge society, 16(4), 6-23.

Garófano, V. V., \& Guirado, L. C. (2017). Importancia de la motricidad para el desarrollo integral del niño en la etapa de educación infantil. EmaisF: revista digital de educación fisica, (47), 89-105. https://dialnet.unirioja.es/servlet/ articulo?codigo $=6038088$

Gaviria-Cortés, Didier Fernando, \& Castejón-Oliva, Francisco Javier (2019). ¿Qué aprende el estudiantado de secundaria en la asignatura de educación física? Revista Electrónica Educare, 23(3), [fecha de Consulta 1 de Junio de 2020]. ISSN: Disponible 
en: https: / / www.redalyc.org/articulo.oa?id=1941/ 194161290002

Grasso, A., \& Erramouspe, B. (2005). La corporeidad escuchada. Revista Novedades Educativas, (175).

Gómez Smyth, L. (2017). La construcción de instancias de juego con rasgos lúdicos en la educación física del nivel inicial. Sportis. Scientific Journal of School Sport, Physical Education and Psychomotricity, 3(3), 569-588. https://doi.org/10.17979/ sportis.2017.3.3.2069

González García, J. (2015). Dramatización y educación emocional. CPU-e, Revista de Investigación Educativa, O(21), 98119. Doi: https://doi.org/10.25009/cpue.v0i21.1723

González Correa, Aída María, \& González Correa, Clara Helena (2010). Educación Física Desde La Corporeidad Y La Motricidad. Revista Hacia la Promoción de la Salud, 15(2), 173187. [Fecha de Consulta 12 de Mayo de 2020]. ISSN: 0121 7577. Disponible en: https://www.redalyc.org/ articulo.oa?id=3091/309126694012

Hurtado Herrera, Deibar Rene(2008). Corporeidad y Motricidad. Una forma de mirar los saberes del cuerpo. Educação \& Sociedade, 29(102), 119-136. [Fecha de Consulta 12 de Mayo de 2020]. ISSN: 0101-7330. Disponible en: https:// www.redalyc.org/articulo.oa?id=873/87314211007

Johnson, R., (2008). Estadistica elemental/Just the Essentials of Elementary Statistics: Lo esencial / The Essentials. Cengage Learning Editores.

Mamani Ramos, A., Dextre Mendoza, C., Lava Gálvez, J., Ticona Flores, G., Quispe Cruz, L., Torres Cruz, F., Quisocala Mamani, J., \& Fuentes López, J. (2020). Desarrollo motor grueso en preescolares de las islas del lago Titicaca (3810 m s. n. m.), Puno, Perú (Gross motor development in preschoolers on the islands of LakeTiticaca (3810 m.a.s.l.), Puno, Peru). Retos, 39, 592-597. https://doi.org/10.47197/ retos.v0i39.79522

Mori, H. R. (2008). La coordinación y motricidad asociada a la madurez mental en niños de 4 a 8 años. Revista Psicología, 16(1), 139-154.

Osorio-Rivera,Verónica Gabriela, Piquer, Marc Pallares, Bartoll, Óscar Chiva, \& Capella-Peris, Carlos. (2019). Efectos de un programa de actividad física integral sobre la motricidad gruesa de niños y niñas con diversidad funcional. Revista Lasallista de Investigación, 16(1), 37-46. https://dx.doi.org/10.22507/ rli.v16n1a2

Parsons, T. (2009). Autobiografía intelectual: elaboración de una teoría del sistema social. Universidad Nacional de Colombia.

PeñaTroncoso, S., ToroArévalo, S., Osses Bustingorry, S., Pachón Moreno, J., \& Hernández Mosqueira, C. (2018). La Dimensión Conceptual del Conocimiento en Educación Física: Un estudio Binacional (Conceptual Dimension of Knowledge in Physical Education: A Binational Study). Retos, (35), 170175. https://doi.org/10.47197/retos.v0i35.63164

Pérez Constante, M. B. (2017). Habilidades del área motriz fina y las actividades de estimulación temprana. Revista Publicando, 4(11(1), 526-537. Recuperado a partir de https:// revistapublicando.org/revista/index.php/crv/article/ view/581

Pérez Samaniego, V., \& Devís Devís, J. (2003). La promoción de la actividad física relacionada con la salud: la perspectiva de proceso y de resultado.

RodríguezAceituno, P., \& Hernández Pina, F. (2017). Didáctica de la Motricidad en la formación de profesores de educación infantil (Didactics of Motricity in early childhood education teachers training). Retos, O(34), 25-32. Recuperado de https: / /recyt.fecyt.es/index.php/retos/article/view/58416

Rojas, M., Bernate, J., Fonseca, I., \& Betancourt, M. (2019) El método praxeologico como herramienta pedagógica de aprendizaje motriz. ATHLOS Revista Internacional de Ciencias Sociales de la Actividad Física, el Juego y el Deporte International Journal of Social Sciences of Physical Activity, Game and Sport, (18)3, 12-35. Recuperado de: https: / / dialnet.unirioja.es/servlet/ articulo?codigo $=7086787$

Ruiz-Pérez, Luis M., \& Barriopedro-Moro, María Isabel, \& Ramón-Otero, Irene, \& Palomo-Nieto, Miriam, \& Rioja-Collado, Natalia, \& García-Coll,Virginia, \& Navia-Manzano, JoséA. (2017). Evaluar la Coordinación Motriz Global en Educación Secundaria: ElTest Motor SportComp. RICYDE. Revista Internacional de Ciencias del Deporte, XIII (49),285-301. [fecha de Consulta 1 de Junio de 2020]. ISSN: 1885-3137. Disponible en: https: / / www.redalyc.org/articulo.oa?id=710/ 71051616007

SailemaTorres, Á., Sailema Torres, M., Amores Guevara, P., Navas Franco, L., Mallqui Quisintuña, V., \& Romero Frómeta, E. (2019). Juegos tradicionales como estimuladores motrices en niños con síndrome de Down. Revista Cubana de Investigaciones Biomédicas, 36(3). Recuperado de http:// www.revibiomedica.sld.cu/index.php/ibi/article/view/72

Salazar Quinatoa, M., \& Calero Morales, S. (2019). Influencia de la actividad física en la motricidad fina y gruesa del adulto mayor femenino. Revista Cubana de Investigaciones Biomédicas, 37(3). Recuperado de http: / /www.revibiomedica.sld.cu/ index.php/ibi/article/view/127

Shusterman, R. (2008). Body consciousness: A philosophy of mindfulness and soma esthetics. Cambridge University Press.

Urrea, P., Bernate, J., Fonseca, I., \& Martínez, A. (2018). Impacto social de los graduados de la Licenciatura en Educación Física, Recreación y Deporte UNIMINUTO. Educación FísicaY Ciencia, 20(2), e050. https://doi.org/10.24215/ $23142561 \mathrm{e} 050$

Valencia-Peris, A., \& Lizandra, J. (2018). Cambios en la representación social de la educación física en la formación inicial del profesorado (Changes in the social representation of physical education during the initial teacher training). Retos, $O(34)$, 230235. Recuperado de https://recyt.fecyt.es/index.php/retos/article/view/60144

Toro-Arévalo, Sergio. (2010). Corporeidad y Lenguaje: La acción como texto y expresión. Cinta de moebio, (37), 44-60. https: / /dx.doi.org/10.4067/S0717-554X2010000100004 